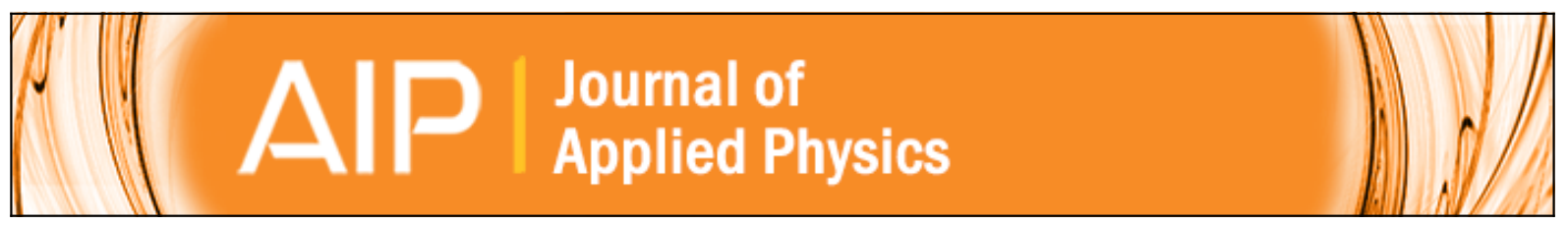

\title{
Trends of hybridization in correlated electron magnetism (invited)
}

B. R. Cooper, J. M. Wills, N. Kioussis, and Q.-G. Sheng

Citation: Journal of Applied Physics 64, 5587 (1988); doi: 10.1063/1.342290

View online: http://dx.doi.org/10.1063/1.342290

View Table of Contents: http://scitation.aip.org/content/aip/journal/jap/64/10?ver=pdfcov

Published by the AIP Publishing

\section{Articles you may be interested in}

Hybridization-induced magnetism in correlated cerium systems

J. Appl. Phys. 79, 6420 (1996); 10.1063/1.361957

Consequences of competing hybridization for magnetic ordering in correlated-electron lattices

J. Appl. Phys. 76, 6130 (1994); 10.1063/1.358329

Prediction of pressure-induced changes in magnetic ordering of correlated-electron uranium systems (invited)

J. Appl. Phys. 75, 7035 (1994); 10.1063/1.356718

First principle evaluation of hybridization and exchange effects for magnetic ordering in correlated-electron uranium systems

J. Appl. Phys. 70, 6083 (1991); 10.1063/1.350053

Trends of hybridization-induced magnetism in cerium monochalcogenides

J. Appl. Phys. 69, 5475 (1991); 10.1063/1.347970

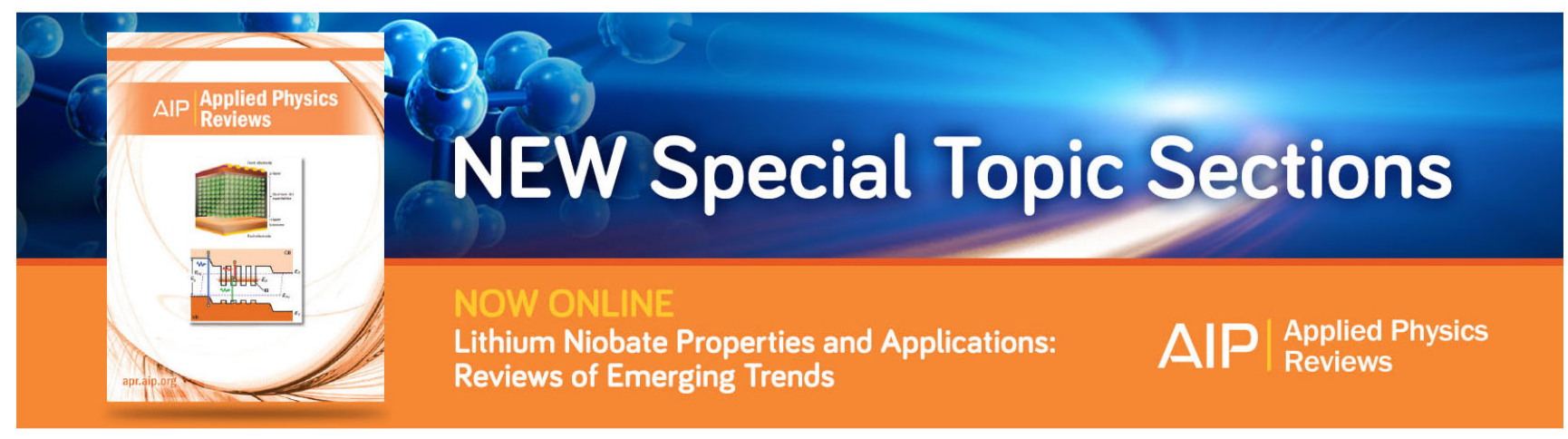




\title{
Trends of hybridization in correlated electron magnetism (invited)
}

\author{
B. R. Cooper \\ Department of Physics, West Virginia University, Morgantown, West Virginia 26506 \\ J.M. Wills \\ Los Alamos Laboratory, Los Alamos, New Mexico 87545 \\ N. Kioussis \\ Department of Physics, California State University, Northridge, California 91330 \\ Q.G. Sherig \\ Department of Physics, West Virginia University, Morgantown, West Virginia 26506
}

\begin{abstract}
Over the past decade, we have: (1) developed phenomenological theory for the behavior of "well-ordered" magnetic states of moderately delocalized light rare-earth and actinide systems (characteristically obtaining unusual anisotropic magnetism in agreement with experiment); (2) developed theory and computational technique to synthesize first principles electronic structure information into that phenomenological theory to make it materially predictive. As discussed in this paper, the resulting theory allows us to predict the triggering of an instability from unusual anisotropic, but well-ordered, magnetism to an unstable state. The unstable state cain be either of a valence fuctuation type or of what probabiy is a heavy fermion type, and the cetailed way in which these two types of instability is triggered differs.
\end{abstract}

\section{NTRODUCTON}

As the felectron atomic shells are filled in the rare earths $\left(4 f^{\prime} s\right)$ and actinides ( $\left.5 f^{\prime} s\right)$, localization of the $f$ electrons in space and energy occurs with increasing atomic number. For the rare earths, this transition to localized behavior occurs on increasing atomic number beyond that of neodymium. However, the degree of delocalization for the lighter rare earths (cerium, praseodymium, neodymium) depends on the detailed chemical environment (specific compound or alloy), and the degree and symptoms of partial delocalization are typically most obvious for cerium mater:als. For the actinides, the transition to localized $f$ behavior occurs on going from piutonium to americium. Again, the degree of $f$ delocalization for the light actinides (uranium, neptunium, plutonium) depends on the specific chemical environment. However, for the same chemical environment (same compound except for change of actinide), one expects considerably more delocalization for uranium than for plutonium. The important thing is that there is a ransitional regime with a range of partially delocalized behavior between itinerant (band or bonding) and localized (correlated ionic) felectron behavior. In recent years there has been much interest in understanding the solid-state behavior characteristic of this transitional regime. Magnetic behavior has played a central role in that interest, both as offering phenomena of interest in themselves and as providing a diagnostic tool to understand how to treat electronic-structure-based behavior generally in the partially delocalized regime of behavior.

Over the past decade, we have: (1) developed phenomenological theory ${ }^{1-10}$ for the behavior of "well-ordered" magnetic states of moderateiy delocalized light rare-earth and actinide systems (characteristically obtaining unusual anisotropic magnerism in agreement with experiment); (2) developed theory and computational technique ${ }^{11,12}$ to synthesize first principles electronic structure information in to that phenomenological theory to make it materially predictive. As discussed below, the resulting theory allows us to predict the triggering of an instability from unusual anisotropic, but well-ordered, magnetism to an unstable state. The unstable state can be either of a valence fuctuation type or of what probably is a heavy fermion type, and the detailed way in which these two types of instability is triggered differs.

The key to understanding solid-state behavior in the transitional regime between localized and delocalized felectron behavior is that the $f$ electrons belonging to one atom feel those belonging to another atom primarily through hybridization (mixing) with band electrons of non $f$ atomic parentage. There are wo main themes involved in our theory for hybridization driven magnetic behavior and instabilities, corresponding to the theory of the magnetic phenomenology and to the synthesis of electronic structure information into that theory, respectively. The crucial point of the phenomenology is that partially delocalized felectron systems have orbitally ariven magnetism. The dominant magnetic coupling is between orbital moments via felectron-band electron hybridization (mixing). This gives a parallel channel to conventional spin-driven magnetism, and this orbitally driven channel dominates the magnetic behavior as the $f$ electrons move from localized (gadoliniumlike) behavior to partially delocalized (ceriumlike) behavior. With regard to fitting band structure (material-specific) information into the phenomenological treatment which in- 
cludes on-site correlation, it is important to develop the phenomenological (model Hamiltonian) tineory and electronic structure theory (used to evaluate parameters of the phenomenological theory) in a unified way. Our phenomenological theory is a resonant scattering theory ${ }^{10,13}$; and our electronic structure theory ${ }^{11,12}$ is a full-potential linearized muffin-tin orbital theory, which is based on a multiple scattering formalism, so that the two segments of theory fit together naturally.

Strongly anisotropic magnetic phenomena are characteristic of the hybridizing (moderately delocalized) regime of behavior. ${ }^{1-3}$ The phenomenological (model Hamiltonian) theory as developed over the past decade has been very successful in explaining a large number of such phenomena in light rare-earth and actinide systems. Among this variety of experimental behavior, there are three key experimental results that shaped or confirm the conceptual nature of the theory. The first of these is the seminal work of Vogt and coworkers ${ }^{14,15}$ involving high field anisotropic magnetization experiments on $\mathrm{CeSb}$ and $\mathrm{CeBi}$, giving extreme anisotropy that is the "wrong way around" (reversed) from that expected for single-ion crystal-field anisotropy; and that furthermore by diuting the cerium with an appropriate divent (yttrium and/or lanthanum), one obtained the anisorropy behavior expected for single-ion crystal-field anisotropy. This showed the presence of an extremely anisotropic two-ion interaction. Second was the neutron inelastic scattering results of Birgeneau $e t a t{ }^{16}$ showing that the crystal-field splitting for $\mathrm{CeSb}$ and $\mathrm{CeBi}$ is an order of magnitude less than that expected by smooth extrapolation of the behavior of the heavier rare-earth and monopnictides. Third, and much more recent, ${ }^{2,3}$ is the recognition that the magnetic ordering temperatures of the light rare-earth antimonides and bismuthides are much higher than would be expected in comparison to the behavior for the gadolinium and heavy rareearth compounds. The first of these results can be understood as coming from a hybridization-mediated twoion interaction; the second can be understood on the basis of hybridization dressing of the crystal feld; and the thiro result can be understood on the basis of hybridization giving rise to coupling between orbital ionic moments (i.e., orbitally driven magnetism).

\section{THEORY AND METHODOLOGY}

Our understanding is based on the Anderson model Hamiltonian for a lattice of $f$-electron ions hybridizing with a band sea. The Hamiltonian then consists of terms for band and localized-f energies with a correlation energy cost $(U)$ to add or subtract an $f$-electron from the nominal $f$ configuration of the ions, and a hybridization term which mixes band and $f$ electrons. By performing $a$ fourth-order Schrieffer-Wolf transformation," the hybridization is transformed to resonant scattering of band electrons of $f$ electrons. This resonant scattering is equivalent to a virtual configuration transition for the $f$-electron ion. This gives rise both to a modification of the felectron ionic behavior (hybridization dressing of the crystal field) and to two-ion interactions via correlated configuration transitions on two $f$ electron ions (two-ion interaction via cooperative hybridization with the band sea). The characteristic parameter giving the single-site band- $f$ hybridization (resonant scattering) strength is called $\mathscr{f}\left(k, m, n, k^{\prime}, m^{\prime}, n^{\prime}\right)$ where $k$ labels band states, $m$ labels $f$ states, and $n$ labels the nominal $f$ ion configuration (e.g., $f^{1}$ for $\mathrm{Ce}^{3+}$ ). $\mathscr{F}$ goes as the square of $V_{k m}$, the band $-f$ hybridization potential matrix element, and inversely as energy differences of the band $\left(\epsilon_{k}\right), f$-electron $\left(E_{m}\right)$, and correlation $(n U)$ energies.

The two-ion hybridization-mediated exchange interaction is given essentially as a product of elements of $\mathscr{J}$ that are off-diagonal in $k$; while the crystal-field dressing goes linearly as elements of $\mathscr{J}$ diagonal in $k$.

Two things have to be done to build a unified phenomenological/electronic structure theory. First, it is necessary to calculate the hybridization potential matrix elements on an absolute basis. Having both the phenomenological and the electronic structure theory in scattering language allows this to be done. Second, to the extent needed, it is necessary to simplify the band structure theory and the phenomenological theory sufficiently to see the physical nature of the dominant physical effect to be evaluated, and then "go backwards" to feed in the full complexity. Doing this is relatively easy for the crystal-field dressing, but is nontrivial for the two-ion interaction that is at the heart of the phenomenology, and involves reducing the size of the two-ion exchange matrix $\mathscr{S}\left(m_{2}^{\prime}, \mathrm{m}_{2} ; m_{1}^{\prime}, m_{1}\right)$ to obtain initial physical understanding before inserting the full complexity of $\mathscr{E}\left(m_{2}^{\prime}, m_{2}\right.$; $\left.m_{1}^{\prime}, m_{1}\right)$ into the absolute theory for the phenomenology.

To understand the second of these points, consider a $\mathrm{Ce}^{3+}$ lattice hybridizing with a band sea. In general, for a given neighbor distance, the two-ion interaction matrix, $\mathscr{C}\left(m_{2}^{\prime}, m_{2} ; m_{1}^{\prime}, m_{1}\right)$ would have $64 / 4$ independent components (reduction by factor of 4 comes from time reversal symmetry). To gain plysical feeling for what is going on depended on reducing the number of independent components per neighbor shell. (When we actually calculate, at present we can keep all $6^{4} / 4$ components when we so desire.) If one has (approximate) axial symmetry about the two-ion axis, one conserves the component of angular momentum along the axis in transitions, and this reduces the number of independent parameters for a $\mathrm{Ce}^{3+}$ lattice to $6^{2} / 4=9$. In mean field theory, hermiticity reduces this to 6 , and if we take the large separation limit this is reduced further to a single component for each neighbor shell, namely that involving $m, m^{\prime}= \pm \frac{1}{2}$ with respect to the two-ion axis. These are the dominant components because they involve $m_{l}=0$ components for the two ions, i.e., the cooperative hybridization effect tends to "suck out" ionic charge along the interionic axis. This then gives the picture of orbitally driven anisotropic two-ion exchange interactions. (Anisotropic, because for $\mathrm{Ce}^{3+}$ the ionic orbital moments tend to align perpendicular to the interionic axis because the hybridized disklike ionic charge cloud wants to have the interionic axis lying in the plane of the disks. For light actinide ions involving more than one $f$ electron this is modified by intraionic correlation.) The experimentally observed sensitivity to the chemical environment can be easily understood when the close relationship between $f$-electron charge shaping and magnetic behavior is recognized. 
In the past four years we have developed a computational scheme ${ }^{11,12}$ to perform a first principles evaluation of the parameters determining $\mathscr{f}\left(k, m, n ; k^{\prime}, m^{\prime}, n^{\prime}\right)$, i.e., the band, $f$, and correlation energies, and the hybridization potential matrix elements; and this has allowed us to put the phenomenological theory on an absolute basis allowing us to predict changes in observable magnetic behavior on going from one material to another.

This comprtational scheme involves a nonconventional electronic structure calculation. With hybridization suppressed, $f$ states are treated as resonant states in a solid-state environment and then localized at each iteration of a selfconsistent electronic structure calculation. This provides the spatial dependence of self-consistently determined localized $f$ states, band states and energies, and a self-consistently determined hybridization potential, thereby allowing determination of the hybridization potential matrix elements (mixing $f$ and band electrons) which is the key ingredient of the theory. The $f$ and correlation energies (in the sense of the model Hamiltonian) are determined from supercell calculations treating one cerium or actinide ion out of four as a defect differing from the nominal configuration by the addition or subtraction of an electron, and then using linear transition theory applied to the $4 f^{0}, 4 f^{1}$, and $4 f^{2}$ energies thus calculated to obtain the $f$ and correlation energies.

The parameters so calculated are then fed into the Ham. iltonian obtained from the Schriefier-Wolfi uransformation of the Anderson lattice Hamilonian, which has the form of a resonant scattering interaction. The direct scattering gives the shift in crystal-field levels (hybridization dressing), and the exchange scattering gives a highly anisotropic two-ion anistropic exchange interaction. Note that the theory provides two-ion interaction with every neighbor that is fully and absolutely determined both as to angular dependence and magnitude. We then use a mean field theory for this Hamilonian to calculate the observable magnetic behavior.

It is important to realize that the mean field theory here involves quite a sophisticated procedure to look at the partial total energy associated with magnetic ordering, i.e., relative to the primary bonding total energy giving the cohesive energy of the solid. The interaction being represented by its time averaged behavior is a complex multipolar interaction. We have developed a sophisticated numerical technique to find all reasonable possibie metastable states, as well as the ground state, at $T=0$ and to follow these as temperature increases. The iterative self-consistent procedure we have developed allows the system to seek whatever metastable states it wants. The procedure does not require the existence of magnetic ordering and will tell us if state mixing (i.e., of ionic states quantized along directions that do not have to be collinear) leads to no magnetic ordering and a truly nonmagnetic state, i.e., withoul a paramagnetic moment.

\section{IP. RESULTS AND DISCUSSBON}

The physical origin of the crystal-field dressing can be pictured rather easily. Unfilled band states of appropriate $\left(\Gamma_{7}\right.$ and $\left.\Gamma_{8}\right)$ symmetry near the Fermi energy mix with crystal-field states of the same symmetry $\left(\Gamma_{7}\right.$ or $\Gamma_{8}$, respectively) and push them down. Our quantitative analysis ${ }^{11}$ indicates that hybridization between $f$ states and band states throughout the Brillouin zone, rather than just in the vicinity of high symmetry points, is important to understanding the magnitude of the relative shift in the crystal-field levels. The set of degenerate band states belonging to the star of a general point in the irreducible wedge of the Brillouin zone forms a basis for a representation of the cubic group that, like the "regular" representation, contains the $\Gamma_{7}$ representation twice and the $\Gamma_{8}$ representation four times. (Here we are discussing cubic systems such as the $\mathrm{NaCl}$ stucture monopnictides and monochalcogenides.) Hence, with twice the number of states hybridizing with the cerium $4 f \Gamma_{8}$ quartet, we expect the effect on the $\Gamma_{8}$ quartet to be larger than the effect on the $\Gamma_{7}$ doublet by approximately a factor of 2 . Our results confirm this expectation. Both $\Gamma_{7}$ and $\Gamma_{8}$ crystal-field $f$-states are lowered, with the effect on $\Gamma_{8}$ greater than the effect on $\Gamma$, by a factor of 2.45 for $\mathrm{CeBi}, 2.56$ for $\mathrm{CeSb}, 2.16$ for CeAs, and 2.09 for CeP.

Now we will discuss the use of our procedure to analyze the trend betwen materials in hybridization-induced effects, using the behavior of two quantities to provide a gross characterization of what is going on: (1) the calculated $f$-state resonance width, $\Gamma$, characterizing the strength of hybridization pe: appropriate band electron, and (2) the density of states at the Fermi energy, $N\left(\epsilon_{F}\right)$, characterizing the number and character (cerium $d$, anion $p$ ) of band states available for hybridization. We have now applied our ab initio treatment to understand ${ }^{12}$ the strong change in nature of magnetic ordering on going from CeSb to CeTe (going from pnictide to chaicogenide means adding one anion valence $p$ electron) both of which have NaCl. structure with a small decrease in lattice parameter. The key result is the way the $a b$ initio absolute calculation signals the change from a stable well-ordered magnetic system to a system on the verge of an instability (probably to a heavy fermion state). In another application, to $\mathrm{CeN}$, we can see how the existence of an unstable valence system (valence fuctuation system) is signaled. ${ }^{77}$ Finally, we will tabulate and discuss trends in hybridization strength more broadly.

\section{A. Change in bahavior trom CeSh to GeT"}

$\mathrm{CeSb}$ is ${ }^{14}$ an antiferromagnet with almost saturated (free-ion) ordered moments aligned along (100). The Neel temperature is $16.8 \mathrm{~K}$ and the ordered moment at low temperature is $2.06 \mu_{B}$. On the other hand, CeTe is ${ }^{18}$ an antiferromagnet with very small ordered moment aligned along (111). The Neél temperature is only $2.4 \mathrm{~K}$ and the ordered moment at low temperature is only $0.20 \mu_{B}$.

On calculating the electronic structure for $\mathrm{CeSb}$ and CeTe treating the $f$ state as a resonant state, we see that the addition of a electron causes the Fermi level to rise into a region of high $C e s d$ density of states. Thus, the big change in behavior on going from CeSb to CeTe is that the bands dominating the hybridization-induced properties in CeTe are largely due to $f$ hybridization with band states largely derived from $C e 5 d$ states. $^{12}$ The resonance width, $\Gamma$, only increases slighty on going from CeSb to CeTe, from 3.06 to $3.21 \mathrm{mRy}$; but $N\left(\varepsilon_{F}\right)$, the dsnsity of states at the Fermi level increases from 4.38 to $15.56 \mathrm{Ry}^{-1}$ where the increase is 
largely due to the $d$ density of states increase from 1.46 to $11.43 \mathrm{Ry}^{-1}$. This implies a large net increase in hybridization because of the big increase in band states available for hybridization, and when we perform the sequence of calculations summarized in the previous section we find a strong change in the range, size, and signs of the two-ion exchange parameters with the various neighbor ions on going from $\mathrm{CeSb}$ to CeTe. If we treat the two-ion exchange matrix at the highest level of approximation, inchding the large ionic separation limit, as described in the previous section there is only one two-ion exchange parameter per neighbor shell. A calculation at this level of approximation gives a free energy per $\mathrm{Ce}^{3+}$ ion associated with magnetic ordering of $-2.6 \mathrm{~K}$ for CeSb, but only - $0.3 \mathrm{~K}$ for CeTe. This means that CeSb is a well magnetically ordered system, while CeTe is only weskly ordered. Indeed the calculations give an ordered noment close to saturation for CeSb and predict a ratio of Neel temperatures of about 10, and also predict the correct change in direction of ordered moment. The degree of approximation of the two-ion exchange matrix can be improved by eliminating the large ionic separation limit approximation, i.e, level of treatment where there are now six calculated exchange parameters per neighbor shell (remember everything used in the caculations is calculated wholly $a b$ intio). On doing this, the free energy associated with magnetic ordering for CeSb increases further in magnitude to $-3.8 \mathrm{~K}$, i.e., the well-ordered state is "confimed" by improving the degree of approximation. However, for CeTe, there is now no stable magnetically ordered state at $T=0$. Thus, the increase in band-f hybridization places CeTe at the borderline between magnetic and nonmagneric behavior in contrast to almost fully ordered CeSb.

\section{B. Prediction of valence fluctuation behavior}

As discussed in the previous section, the f-state and correlation energies, $E_{f}$ and $U$, are obtained by performing a sequence of three supercell calculations (unit cell equal to four normal unit cells) treating one cerium as a defect having $f^{\circ}$ or $f^{2}$ configuration (as well as a "control" supercell calculation where the defect site ras the same $f$ configuration as the other sites $)$. The values of $E_{f}$ and $U$ are then obtained from the $f^{*}, f^{1}$, and $f^{2}$ energy levels by the use of linear transition theory. For the heavier cerium monopnictides this procedure gives the $4 f^{\prime}$ level about $0.5 \mathrm{eV}$ below the

TABLEI. Calculated resonance widh $\Gamma$, for cerium and plutonium monopnictides and monochalcogenides.

\begin{tabular}{lrlc}
\hline & $\Gamma(m R y)$ & $\Gamma(m R y)$ \\
\hline CeN & 21.22 & & \\
Cep & 4.99 & & \\
CeAs & 4.28 & CeTe & 3.21 \\
CeSb & 3.06 & & \\
CeBi & 4.23 & & \\
PuAs & 19.34 & PuTe & 17.86 \\
PuSb & 10.49 & & \\
PuBi & 10.55 & \\
\hline
\end{tabular}

Fermi level; and $E_{f}$ is about $3 \mathrm{eV}$ below the Fermi level, ${ }^{17}$ with $U$ equal to about $6 \mathrm{eV}$. The resonance width, $\Gamma$, for the heavier cerium monopnictides varies between a low value of about $3 \mathrm{mRy}$ for CeSb to a high value of about $5 \mathrm{mRy}$ for CeP. For CeN, the value of $\Gamma$ increases ${ }^{17}$ to $21.2 \mathrm{mRy}$; as discussed below this is associated with a large increase of $f$ hybridization with Ce $5 d$ band states. The calculated $4 f^{\prime}$ level moves to $2.1 \mathrm{eV}$ above the Fermi level while the $4 f^{\circ}$ level is $4.8 \mathrm{eV}$ below the Fermi level. When we recognize that the Fermi level is an infinite sink of electrons, this movement of $4 f^{\prime}$ above the Fermi level indicates a valence instability, a fuctuation beween $f^{\prime}$ and $f^{\prime}$; and $\mathrm{CeN}$ is experimentally observed $^{19}$ to be an unstable valence system.

\section{Trends in hybridization indicated by resonance widh}

As shown in Table I, we can use the value of calculated resonance width, $\Gamma$, as one way of characterizing the trend between materials in strength of hybridization. (Remember that band densities of states also indicate changes in hybridization. Also, we expect a decreased calculated correlation energy $U$ as another result of increased hybridization.)

Note the overall increase in $\Gamma$ on going between corresponding cerium and plutonium compounds. This corresponds to greater $f$ delocalization, hence increased hybridization. We expect another significant increase on going to uranium.

For the cerium monopnictides note the minimum in $\Gamma$ at CeSb. For the lighter cerium monopnictides (CeN$\mathrm{CeSb}$ ), the lattice parameter is of a size that just accommodates cerium and pnictogen atoms of "typical solid-state size"; while for CeBi the lattice parameter is too small to do this. Thus, the increase of hybridization on going up the pnictogen column from the antimonide has a simple explanation in increased $f$ hybridization with $5 d$ lectrons on other cerium sites as the cerium-cerium separation decreases with decreasing anion size; the decrease on going to the nitride is large, giving a large increase in hybridization. On the other hand, the increase in $\Gamma$ on going from $\mathrm{CeSb}$ to $\mathrm{CeBi}$ corresponds to the increased $\mathrm{C} f \mathrm{f}$ hybridization with anion valence $p$ electrons as the cerium and bismuth draw closer together than would be expected from simple solid-state atomic size considerations.

It is well to remember that Table 1 does not show the large increases in hybridization that can be associated wuith density of stares efrects. As discussed in Sec. III A above, such effects drive CeTe to the edge of magnetically unstable behavior. It is very interesting that $P$ Te is the most anomalows material in Table I, probabiy being a heavy fermion system. $^{20-22}$ In pure we have a large increase in resonance width from $\mathrm{PuSb}$, and in adcition we expect a large increase in density of states of the same sort as occurs between CeSb and CeTe.

In conclusion, we believe we have developed a computational technique synthesizing model Famitonian and eiectronic structure theory that reliably allows us to understand and predict which light rare-earth and light actinide materials should have hybridization-driven magnetic and valence instabilities. 


\section{ACKNOWEEDGERETS}

This research was supported by National Science Foundation Grant No. DMR-85-04449. The research at Los Alamos National Laboratory was supported by the U.S. Dept. of Energy. Suggestions by and discussions with H. R. Ott and J. Rossat-Mignod were very valuable in guiding and understanding the calculations discussed in the final section of this paper.

'B. R. Cooper, R. Sieman, D. Yang, $P$. Thayamballi, and A. Banerjea, Handbook an the Physics and Chemistry of the Actinides, edited by A. I. Freeman and G. H. Lander (North-Holland, Ansterdam, 1985), Vol. 2, Chap. 6, pe. 435-500.

3. R. Cooper, J. Less-Common Met. 133, 3: (1987).

${ }^{3}$ B. R. Cooper, G. J. Hu, N. Kioussis, and J. M. Wills, J. Magn. Magn. Mater. 63, 12! (1987).

${ }^{4}$ B. R. Cooper and R. S. Siemann, J. Appl. Phys. 50, 1991 (1979); R. Siemann and B. R. Cooner, Phys. Rev. Lett. 44, 1015 (1980).

${ }^{5}$ P. Thayamballi and B. R. Cooper, J. Appl. Phys. 55, 1829 (1984).

${ }^{\circ}$ A. Banerjea and B. R. Cooper, Phys. Rev. B 34, 1607 (1986).

${ }^{7}$ N. Kioussis and B. Cooper, Phys. Rev. B 34, 3261 (1986).
N. Kioussis, B. R. Cooper, and A. Banerjea, J. Appl. Phys. 61, 3388 (1987); to appear in Phys. Rev. B (1988).

${ }^{9} \mathrm{G}$. J. Hu and B. R. Cooper, J. Appl. Phys. 63, 3826 (1988); F. Thayamballi and B. R. Cooper, Phys. Rev. B 31, 5911 (1985).

${ }^{10} \mathrm{G}$. J. Hu, N. Kioussis, A. Banerjed, and B. R. Cooper, Phys. Rev. B 38 , 2639 (1988); G. I. Hu and F. R. Cooper, to appear in Phys. Rev. B (1988).

"J. M. Wils and B. R. Cooper, Phys. Rev. B 36, 3809 (1987).

${ }^{13}$ N. Koussis, B. R. Cooper, and J. M. Wills, J. Appl Phys. 63, 3683 (1988).

${ }^{13}$ B. Coqbin and J. R. Schriefier, Phys. Rev. 185, 847 (1969).

${ }^{14} \mathrm{G}$. Busch and O. Vogt, Phys. Lett. 25 $\mathrm{B}, 449$ (1967).

${ }^{15}$ B. R. Cooper and O, Vogt, J. Phys. (Paris) Collog. 32, Cl-1026 (1970).

${ }^{\prime \prime R}$ R. J. Rirgenean, E. Bucher, J. P. Maita, L. Passeli, and K. C. Tuberfeld, Phys. Rev. 18 8, 5345 (1973).

17Q-G. Sheng. B. R. Cooper, N. Kioussis, and J. M. Wills, Bull. Am. Phys. Soc. 33, 364 (1988).

${ }^{18} \mathrm{~F}$. Hulliger, B. Natterer, and H. R. Otr, J. Magn. Magn. Mater. 8, 87 (1978); R. R. Ot, J. K. Kjems, and F. Jlulliger, Phys. Rev. Lett. 42, 1378 (1979).

${ }^{19} \mathrm{Y}$. Baer and $\mathrm{Ch}$. Zurcher, Mhys. Rev. Lett. 39, 956 (1977).

${ }^{20} \mathrm{~K}$. Mattenberger, O. Vogt, J. C. Spirlet, and J. Rebizant, J. Less-Conmon Met. 21, 285 (1986).

21 J. M. Collard, A. Blaise, J. M. Fournier, and J. P. Charvillat, J. LessCommon Met. 121, 223 (1986).

${ }^{22 \mathrm{~g} . ~ G . ~ T h e r o n d, ~ A . ~ B l a i s e, ~ J . ~ M . ~ F o u r n i e r, ~ J . ~ R o s s a t-M i g n o d, ~ J . ~ C . ~ S p i r l e t, ~}$ J. Rebizant, and O. Vogt, J. Magn. Magn. Mater. 63, 142 (1987). 